\title{
Silencing CDCA8 inhibits the proliferation and invasion of gastric cancer cells and induces apoptosis by blocking the Akt pathway
}

\author{
Fuxiang Fan, Jingbo Du, Yanbo Lou* \\ General Surgery, The Fourth Affiliated Hospital Zhejiang University School of Medicine, Yiwu, Zhejiang Province 322000, China
}

*For correspondence: Email: yanbo_lou@zju.edu.cn; Tel: +86-057989935894

Sent for review: 10 November 2020

Revised accepted: 25 February 2021

\begin{abstract}
Purpose: To investigate the effect of cell division cycle associated 8 (CDCA8) on malignant progression of gastric cancer (GC) cells.

Methods: Short hairpin RNAs (shRNA) were transfected into two gastric cell lines to knock down expression of CDCA8. Transfection efficiency was analyzed using quantitative real-time polymerase chain reaction (qRT-PCR) and western blot. Then, Cell Counting Kit 8 and colony formation and Transwell assays were utilized to explore the effect of CDCA8 knockdown on the proliferation, invasion, and migration of GC cells. Flow cytometry was conducted to analyze the effect of CDCA8 knockdown on cell cycle progression and apoptosis. The relationship between CDCA8, epithelial-mesenchymal transition (EMT), and Akt pathway activity was determined by western blot analysis.

Results: Proliferation, invasion, and migration of GC cells were significantly inhibited by CDCA8 knockdown. Knockdown of CDCA8 induced cell cycle arrest in G1 phase and apoptosis, and inhibited EMT and Akt pathway activity.

Conclusion: Knockdown of CDCA8 inhibits GC growth and metastasis in vitro by reducing Akt pathway activity. Thus, this molecule presents a potential strategy for the management of GC
\end{abstract}

Keywords: CDCA8, Gastric cancer, Akt signaling pathway, Proliferation, Metastasis

\begin{abstract}
This is an Open Access article that uses a fund-ing model which does not charge readers or their institutions for access and distributed under the terms of the Creative Commons Attribution License (http://creativecommons.org/licenses/by/4.0) and the Budapest Open Access Initiative (http://www.budapestopenaccessinitiative.org/read), which permit unrestricted use, distribution, and reproduction in any medium, provided the original work is properly credited.

Tropical Journal of Pharmaceutical Research is indexed by Science Citation Index (SciSearch), Scopus, International Pharmaceutical Abstract, Chemical Abstracts, Embase, Index Copernicus, EBSCO, African Index Medicus, JournalSeek, Journal Citation Reports/Science Edition, Directory of Open Access Journals (DOAJ), African Journal Online, Bioline International, Open-J-Gate and Pharmacy Abstracts
\end{abstract}

\section{INTRODUCTION}

Gastric cancer (GC) is one of the most aggressive common malignancies, with approximately 1,000,000 new cases and 781,000 deaths according to global cancer statistics in 2018 [1]. GC has the second highest morbidity and mortality in China, with 679,100 new cases and 49,800 deaths in 2015 [2]. GC progresses rapidly to advanced stages, and the high recurrence rate and high invasiveness of advanced GC are the main causative factors of patient deaths. The high invasiveness of GC is also the main obstacle to improving patients' survival rate after surgical resection [3].

The mechanisms that drive cancer occurrence and metastasis include those that promote proliferation, migration, invasion, and angiogenesis, and lead cells to evade aging and 
death $[4,5]$. Cell division cycle associated 8 (CDCA8) is a component of vertebrate chromosomal passenger complex (CPC), and the overexpression of CPC is associated with the occurrence of cancer [6-8].

CDCA8 targets CPC components to the centrosome, corrects kinetochore attachment errors, and stabilizes the bipolar axis of human cells, and thus has important regulatory functions during mitosis $[6,9,10]$. Overexpression of CDCA8 is associated with malignant progression of cancer and poor patient prognosis in a variety of human cancers, including pancreatic ductal adenocarcinoma [11] and lung cancer [12]. Therefore, CDCA8 is considered a potential new therapeutic target and prognostic marker for cancer.

It has been reported that abnormal expressions of chromosomal guest proteins that regulate mitosis is associated with poor prognosis of GC [13], but the role of CDCA8 in GC is not clear. Our study investigates whether the aberrant expression of CDCA8 affects the malignant progression of $\mathrm{GC}$ and, if it does, its potential mechanism of action. Our overall goal is to provide new effective therapeutic targets for advanced GC.

\section{EXPERIMENTAL}

\section{Cell culture and transfection}

The GC cell lines SGC-7901 (CL-0206) and AGS (CL-0022) were obtained from Procell Life Sciences (Wuhan, China), and cultured in RPMI 1640 medium (Thermo Fisher Scientific, MA, USA) containing $10 \%$ fetal bovine serum (FBS) (Gibco) at $37^{\circ} \mathrm{C}$ in an incubator containing $5 \%$ $\mathrm{CO}_{2}$ humidified air. The pLKO.1 vector and CDCA8 shRNA were acquired from Addgene (Watertown, MA, USA). The CDCA8 shRNA was cloned into the pLKO.1 vector and lentiviral transfection was used to construct SGC-7901 and AGS cell lines with stable knockdown of CDCA8 expression.

\section{Quantitative real-time polymerase chain reaction (qRT-PCR)}

Total RNA was extracted from GC cells using TRIzol reagent (Life Technologies, Thermo Fisher Scientific) according to the manufacturer's instructions. One $\mu \mathrm{g}$ of total RNA was reverse transcribed into cDNA using reverse transcriptase and Oligo dT primers (Takara, Japan). The cDNA was then amplified via PCR using specific primers. The CDCA8 primers used for PCR were 5"-GTTCCAAGGAGAAGCCC
ACA-3` (forward) and 5`-CCGGTCCACATTC TCTGGAA-3" (reverse). The primers used to amplify $\beta$-actin as internal control were $5^{\circ}$ CATGTACGTTGCTATCCAGGC-3` (forward) and 5'-CTCCTTAATGTCACG CACGAT-3 (reverse). Cycle threshold $(\mathrm{Cq})$ values were obtained in each sample and relative expression levels were calculated using the $2^{-\Delta \Delta C t}$ method [14].

\section{Western blot}

GC cells were lysed with lytic buffer (Merck Millipore, USA), and extracted proteins were quantified using the bicinchoninic acid assay. Ten - 20 $\mathrm{\mu g}$ of extracted protein was separated using $12 \%$ SDS-PAGE and transferred to a PVDF membrane (Merck Millipore). After blocking non-specific sites with $5 \%$ skim milk at room temperature for $2 \mathrm{~h}$, the PVDF membrane was incubated overnight at $4{ }^{\circ} \mathrm{C}$ with the appropriate primary antibody dilution as shown in Table 1. Then, the membrane was incubated with a 1:2000 dilution of goat anti-rabbit IgG H\&L (HRP) (ab205718, Abcam) at $20{ }^{\circ} \mathrm{C}$ for $2 \mathrm{~h}$. The immunoreactive proteins were visualized using chemiluminescence (Santa Clara, USA).

Table 1: Primary antibody details

\begin{tabular}{llll}
\hline Protein & Item number & Dilution & Company \\
\hline CDCA8 & ab74473 & $1: 1000$ & Abcam (UK) \\
CyclinD1 & ab134175 & $1: 10000$ & Abcam \\
p21 & ab109520 & $1: 1000$ & Abcam \\
p27 & ab193379 & $1: 1000$ & Abcam \\
Bcl-2 & ab182858 & $1: 2000$ & Abcam \\
Bax & ab32503 & $1: 1000$ & Abcam \\
E-cadherin & ab40772 & $1: 10000$ & Abcam \\
N-cadherin & ab245117 & $1: 1000$ & Abcam \\
p-PI3K & BS4605 & $1: 1000$ & Bioworld (USA) \\
p-Akt & ab8805 & $1: 500$ & Abcam \\
Akt & ab38449 & $1: 500$ & Abcam \\
GAPDH & ab128915 & $1: 10000$ & Abcam \\
\hline
\end{tabular}

\section{Cell proliferation assay}

Cell Counting Kit 8 assay (CCK-8, Dojindo Laboratories, Kumamoto, Japan) was performed to evaluate cell viability. SGC-7901 or AGS cells $\left(1 \times 10^{3}\right)$ were spread in 96-well plates, and incubated for $24 \mathrm{~h}$. CCK-8 reagent was added to the wells and incubation was continued for $1 \mathrm{~h}$, and the absorbance was then measured at 450 $\mathrm{nm}$ using an automatic enzyme labeling instrument (Synergy4, VT, USA).

\section{Colony formation assay}

The cells were digested with trypsin, inoculated into a 6 -well plate at a density of $1 \times 10^{3}$ cells / well, and incubated at $37{ }^{\circ} \mathrm{C}$ for 14 days. Cells were fixed with $20 \%$ methanol solution and later 
stained with $0.1 \%$ crystal violet. The colonies were observed under an inverted microscope, then counted and analyzed.

\section{Cell cycle analysis}

SGC-7901 or AGS cells were placed in $70 \%$ cold ethanol overnight. Ethanol was removed by centrifugation and the cells were incubated gradually with RNase $A(10 \mu \mathrm{g} / \mathrm{ml})$ and propidium iodide $(\mathrm{PI})$ solution $(50 \mu \mathrm{g} / \mathrm{ml})$ at $37^{\circ} \mathrm{C}$. Finally, the DNA content of the cells was analyzed via flow cytometry.

\section{Apoptosis assay}

Apoptosis was analyzed using the FITC Annexin V Apoptosis Detection Kit 1 (BD Pharmingen, CA, USA). Cells in logarithmic phase were inoculated in a 6-well plate at a density of $1 \times 10^{5}$ cells per well and incubated for $24 \mathrm{~h}$. The cells were collected, and washed and stained under dark conditions with Annexin V FITC and PI for 15 min. Cytomics FC 500 (Beckman Coulter, CA, USA) was used for flow cytometric analysis.

\section{Transwell assay}

Invasion and migration assays were performed in transwell chambers pre-coated with or without Matrigel, for invasion and migration, respectively. Briefly, $5 \times 10^{4}$ SGC-7901 or AGS cells were inoculated into the upper chamber which was covered with $200 \mu \mathrm{l}$ serum-free medium. The lower chamber contained $600 \mu \mathrm{L}$ medium and 10 $\%$ FBS added as a chemical attractant. After incubation for $24 \mathrm{~h}$, the cells that had migrated or invaded the lower chamber were fixed with formaldehyde and stained with $0.5 \%$ crystal violet for $5 \mathrm{~min}$. The cells were photographed and counted under an inverted microscope.

\section{Statistical analysis}

The results were statistically analyzed with SPSS 22.0 (IBM Corp., Armonk, NY, USA) software. All values were expressed as mean \pm SEM. The experiments were repeated 3 times independently. Differences between paired samples were analyzed using the t-test and were statistically significant when $p<0.05$.

\section{RESULTS}

\section{Knockdown of CDCA8 inhibits the growth of} GC cells in vitro

CDCA8 is often reported as an oncogene, which suggests that CDCA8 may promote the malignant growth of GC. In order to test this conjecture, a recombinant vector was constructed from shRNA against CDCA8 and transfected into SGC-7901 and AGS cells. CDCA8 shRNA significantly decreased the expression of CDCA8 mRNA and protein in GC cell lines, and pLKO-1\# CDCA8 knockdown had the greatest effect (Figure $1 \mathrm{~A}$ and $\mathrm{B}$ ). Therefore, this shRNA was selected for experimental exploration and named pLKO CDCA8. The CCK8 assay showed that knockdown of CDCA8 expression significantly decreased cell viability (Figure $1 \mathrm{C}$ ). The colony formation test demonstrated that the number of colonies formed by each cell line decreased significantly after knocking down CDCA8 (Figure 1 D). These results show that knockdown of CDCA8 inhibits the growth of GC cells in vitro.
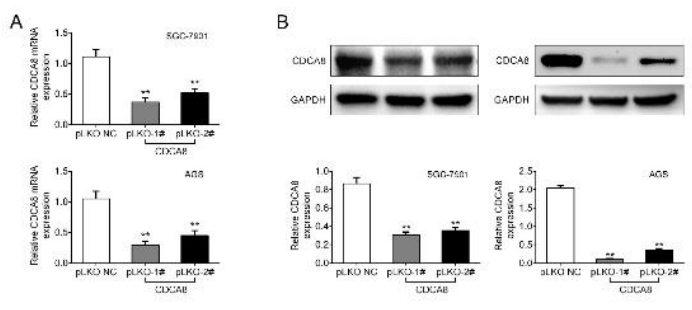

C

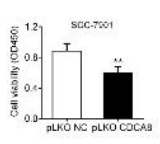

D
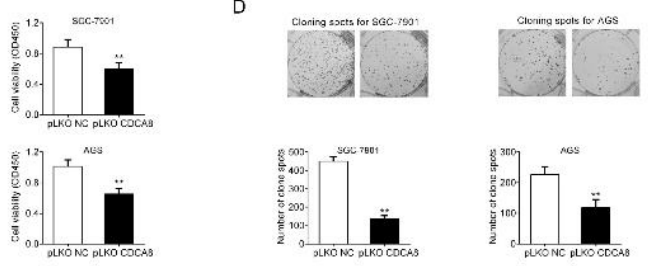

Figure 1: Knockdown of CDCA8 inhibits the proliferation of GC cells in vitro. (A) Effect of shRNA on CDCA8 mRNA expression. (B) Western blot showing the inhibitory effect of shRNA on CDCA8 protein expression. (C) The effect of CDCA8 knockdown on SGC-7901 and AGS cell viability. (D) Colony formation test was used to analyze the colony formation ability of SGC-7901 and AGS cells after knocking down CDCA8; ${ }^{* *} p<0.01$ vs. PLKO NC

\section{CDCA8 knockdown induce cell cycle arrest in GC cells}

CDCA8 regulates the mitotic process of cells and promotes the growth of GC cells in vitro. Therefore, knockdown of CDCA8 may induce cell cycle arrest. This speculation was tested using flow cytometry, which showed that CDCA8 knockdown induced cell cycle arrest in G1 phase (Figure $2 \mathrm{~A}$ ). Furthermore, the protein levels of CyclinD1, p21 and p27 were detected via western blot (Figure $2 \mathrm{~B}$ ). The data showed that after CDCA8 knockdown, the expression of CyclinD1 was significantly decreased, while the expression of cyclin-dependent kinase inhibitors (p21 and p27) was significantly increased. These 
results confirmed that CDCA8 knockdown induced cell cycle arrest.
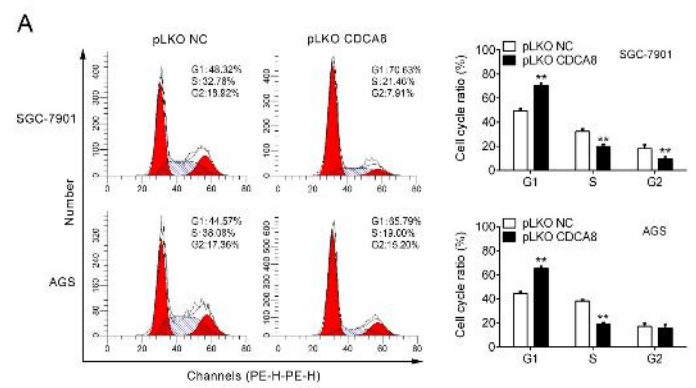

B

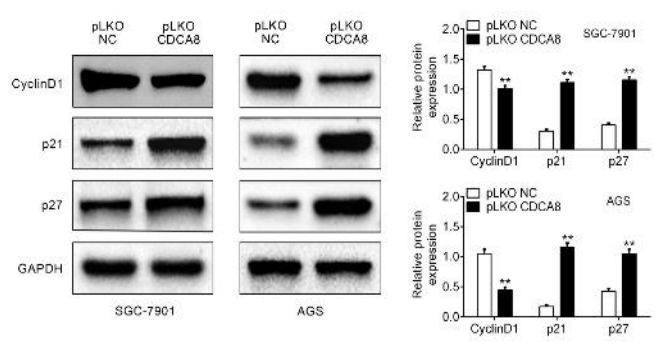

Figure 2: Knockdown of CDCA8 induces cell cycle arrest in vitro. (A) Cell cycle distribution in SGC-7901 and AGS cells after knockdown by CDCA8. (B) Expression of cell cycle related proteins CyclinD1, p21 and p27 in SGC-7901 and AGS cells after CDCA8 knockdown; ${ }^{* *} p<0.01$ vs. PLKO NC

\section{CDCA8 knockdown induces apoptosis in GC cells}

Apoptosis is one of the main pathways that leads to cell growth inhibition. The ratios of apoptotic to non-apoptotic SGC-7901 and AGS cells were determined by flow cytometry. The results showed that knockdown of CDCA8 significantly increased the ratio of apoptotic GC cells (Figure 3 A). Similarly, the expression of apoptosisrelated proteins was analyzed by western blot. After knocking down CDCA8, the expression of the anti-apoptotic protein $\mathrm{Bcl}-2$ was inhibited, while the expression of the pro-apoptotic protein Bax was significantly increased (Figure 3 B). Combined with the results of flow cytometry and western blot analysis, these results confirm that knockdown of CDCA8 significantly induces apoptosis of GC cells.

\section{CDCA8 knockdown inhibit invasion and migration of $\mathrm{GC}$ cells}

To measure the influence of CDCA8 knockdown on cell metastasis, its influence on SGC-7901 and AGS cell invasion and migration was assessed using the transwell assay. As shown in Figure $4 \mathrm{~A}$, knockdown of CDCA8 inhibited invasion and migration of GC cells. In addition, the effect of CDCA8 knockdown on the expression of epithelial-mesenchymal transition (EMT)-related proteins was analyzed using western blotting (Figure $4 \mathrm{~B}$ ). The results showed that the expression of E-cadherin protein in SGC7901 and AGS cells increased, while the expression of $\mathrm{N}$-cadherin decreased after CDCA8 knockdown.
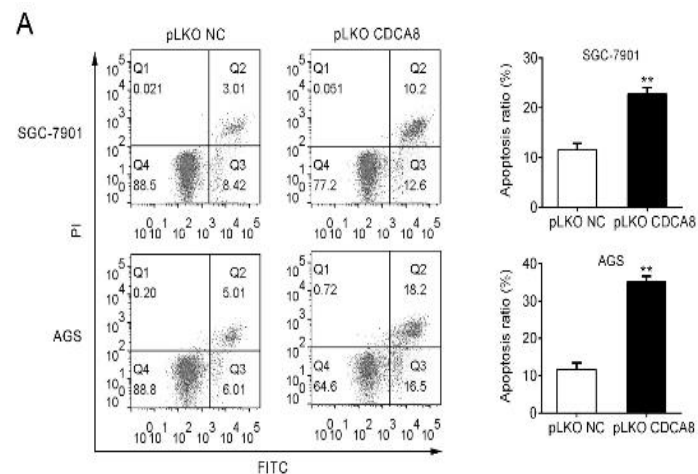

B
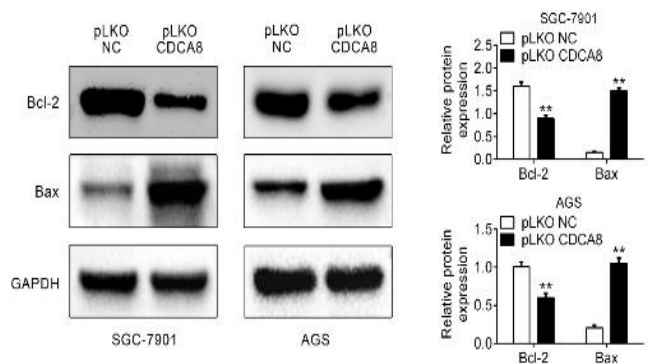

Figure 3: Knockdown of CDCA8 induces apoptosis in vitro. (A) Effect of CDCA8 knockdown on apoptosis in SGC-7901 and AGS cells. (B) Effect of knocking down CDCA8 on the expression of apoptosis-related proteins Bcl-2 and Bax in SGC-7901 and AGS cells; ${ }^{* *} p<0.01$ vs. PLKO NC

CDCA8 knockdown inhibit the activity of Akt signal pathway

Knocking down CDCA8 down-regulated p-PI3K and p-Akt (Figure 5). Therefore, CDCA8 knockdown inhibits the growth and invasiveness of GC cells, at least in part, by inactivating the Akt pathway.

\section{DISCUSSION}

In recent years, research on GC targets and prognostic markers has accelerated. Although no study has reported a role for CDCA8 in the development of $\mathrm{GC}$, its regulation of the mitotic processes [9] and the pro-carcinogenic role it exhibits in common cancers such as lung [12] and pancreatic cancer [11] are noteworthy. 


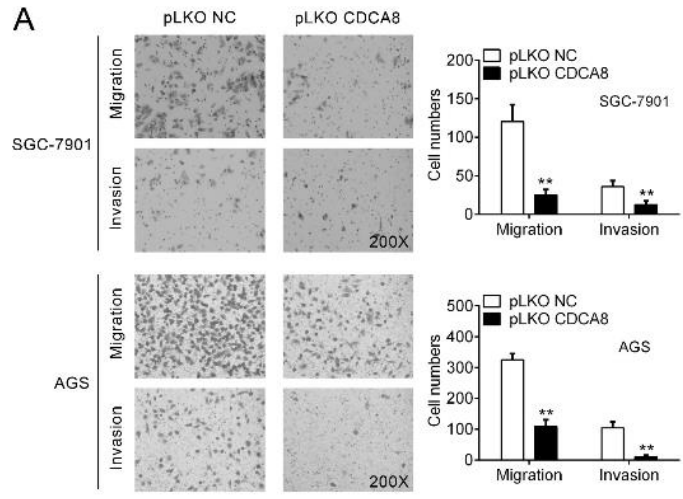

B

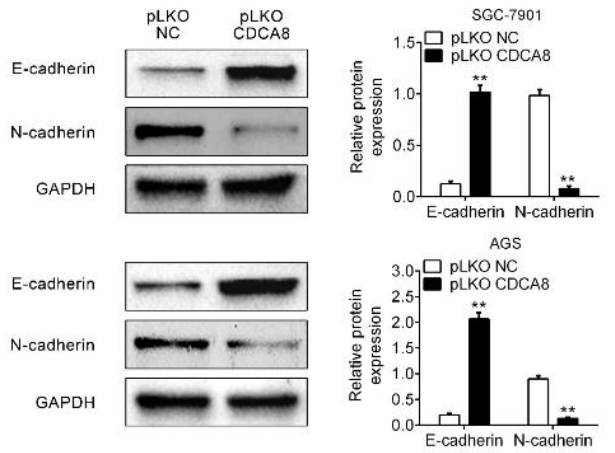

Figure 4: Knockdown of CDCA8 inhibits biological functions related to cell metastasis. (A) Migration and invasion of SGC-7901 and AGS cells with or without knockdown of CDCA8. (B) Effect of knockdown of CDCA8 on the expression levels of the EMT-related proteins E-cadherin and $\mathrm{N}$-cadherin; ${ }^{* *} p<0.01$ vs. PLKO NC

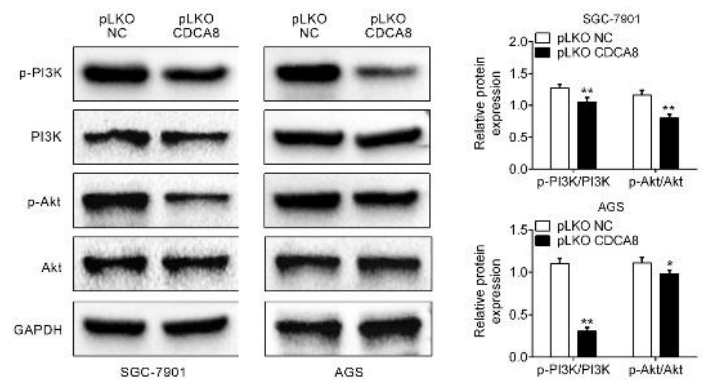

Figure 5: Knockdown of CDCA8 inhibits Akt pathway activity. Effect of knockdown of CDCA8 on the expression and activation of Akt pathway-associated proteins. ${ }^{*} P<0.05,{ }^{* *} p<0.01$ vs. PLKO NC

In terms of cell growth, this study revealed, through CCK-8 and colony formation assays, that knockdown of CDCA8 inhibited GC cell viability and proliferation ability. This result is consistent with previous reports that CDCA8 knockdown inhibited the proliferation of skin melanoma cell lines [17], and bladder [18] and breast cancer
[19]. Can et al [20] found that the CDCA8 promoter has high activity in several cancer cell lines, as well as in undifferentiated human embryonic stem cells (hESCs) and early mouse embryos, which share the common feature of retaining high proliferative activity. This suggests that the activation of CDCA8 transcription contributes to cell growth, and explains the inhibition of GC cell proliferation by CDCA8 knockdown found in this study. Furthermore, the results of flow cytometry demonstrated that knockdown of CDCA8 induced cell cycle G1 arrest and induced apoptosis. As early as 2003, a study on the cell cycle reported that CDCA8 regulates mitosis in cells [10]. In addition, Hayama et al. [12] confirmed that knocking down CDCA8 in lung and ovarian cancer cells can induce cell cycle arrest, which is consistent with the findings of the present work.

This study also revealed that CDCA8 knockdown inhibited the migration and invasiveness of $G C$ cells. CDCA8 may be a key gene involved in the metastasis of clear cell renal cell carcinoma [21] and may promote metastasis of prostate cancer[22] and melanoma [17]. In addition, this study found that knockdown of CDCA8 upregulated $\mathrm{E}$-cadherin and downregulated $\mathrm{N}$ cadherin expression. During EMT, E-cadherin, which is responsible for normal epithelial cell adhesion, is replaced by $\mathrm{N}$-cadherin, which provides better linkage flexibility and enhances cell motility [23]. This suggests that knockdown of CDCA8 inhibits EMT. This is the first report that abnormal expression of CDCA8 affects EMT, not only in the field of GC research, but also in the field of cancer research.

This study further investigated the potential mechanism of CDCA8 knockdown in inhibiting GC progression and found that CDCA8 knockdown led to inactivation of the Akt signaling pathway. Activation of the PI3K/Akt signaling pathway has previously been shown to promote GC occurrence and invasion [24]. Also, inactivation of the PI3K/Akt signaling pathway led to GC cell autophagy and apoptosis [25]. These reports on the association of PI3K/Akt signaling pathway activity with $\mathrm{GC}$ support the conclusion of this study that CDCA8 knockdown inhibits GC growth and metastasis by inducing inactivation of the Akt pathway.

\section{CONCLUSION}

This study is the first to investigate the effect of aberrant expression of CDCA8 on GC progression and revealed that CDCA8 knockdown inhibits GC cell growth and metastasis in vitro by inducing cell cycle arrest 
and Akt pathway inactivation. It fills a critical gap in our understanding of the involvement of CDCA8 in GC and is crucial to assess the value of CDCA8 as a therapeutic target and prognostic indicator in GC. Further research will involve animal experiments to illuminate the effect of CDCA8 on gastric cancer growth and metastasis in vivo.

\section{DECLARATIONS}

\section{Acknowledgement}

This work was supported by Research projects of Yiwu City, Zhejiang Province (Grant no. 20-3064).

\section{Competing interests}

There are no conflicts of interest to disclose.

\section{Contribution of authors}

We declare that this work was done by the authors named in this article and all liabilities pertaining to claims relating to the content of this article will be borne by the authors. Fuxiang Fan designed the study and supervised the data collection; Jingbo Du analyzed and interpreted the data; Yanbo Lou prepared the manuscript for publication and reviewed the draft of the manuscript. All authors have read and approved the manuscript.

\section{Open Access}

This is an Open Access article that uses a funding model which does not charge readers or their institutions for access and distributed under the terms of the Creative Commons Attribution License (http://creativecommons.org/licenses/by/ 4.0) and the Budapest Open Access Initiative (http://www.budapestopenaccessinitiative.org/rea d), which permit unrestricted use, distribution, and reproduction in any medium, provided the original work is properly credited.

\section{REFERENCES}

1. Bray F, Ferlay J, Soerjomataram I, Siegel RL, Torre $L A$, Jemal A. Global cancer statistics 2018: GLOBOCAN estimates of incidence and mortality worldwide for 36 cancers in 185 countries. CA Cancer J Clin 2018; 68(6): 394-424.

2. Chen $W$, Zheng $R$, Baade $P D$, Zhang S, Zeng H, Bray $F$, Jemal A, YU XQ, He J. Cancer statistics in China, 2015. CA Cancer J Clin 2016; 66(2): 115-132.
3. Van Cutsem E, Sagaert X, Topal B, Haustermans K, Prenen H. Gastric cancer. Lancet 2016; 388(10060): 2654-2664.

4. Hanahan D, Weinberg RA. Hallmarks of cancer: the next generation. Cell 2011; 144(5): 646-674.

5. Zhang $Y$, Wang J, LiU X, Li J, Fan S. MicroRNA miR103a-3p targets NPAS3 to regulate progression of Alzheimer's disease. Tropical Journal of Pharmaceutical Research 2020; 19; 1015-1021.

6. Gassmann R, Carvalho A, Henzing AJ, Ruchaud S, Hudson DF, Honda R, Nigg EA, Gerloff DL, Earnshaw WC. Borealin: a novel chromosomal passenger required for stability of the bipolar mitotic spindle. J Cell Biol 2004; 166(2): 179-191.

7. Gerlach U, Kayser G, Walch A, Hopt U, Schulte-Mönting $J$, Werner M, Lassmann S. Centrosome-, chromosomalpassenger- and cell-cycle-associated mRNAs are differentially regulated in the development of sporadic colorectal cancer. J Pathol 2006; 208(4): 462-472.

8. Tanaka K, Iwamoto S, Gon G, Nohara T, Iwamoto M, Tanigawa $N$. Expression of survivin and its relationship to loss of apoptosis in breast carcinomas. Clin Cancer Res 2000; 6(1): 127-134.

9. Liu X, Song Z, Huo Y, Zhang J, Zhu T, Wang J, Zhao X, Aikhionbare $F$, Zhang J, Duan $H$ et al. Chromatin protein HP1 interacts with the mitotic regulator borealin protein and specifies the centromere localization of the chromosomal passenger complex. J Biol Chem 2014; 289(30): 20638-20649.

10. Higuchi T, Uhlmann F. Cell cycle: passenger acrobatics. Nature 2003; 426(6968): 780-781.

11. Li B, LiU B, Zhang X, LiU H, He L. KIF18B promotes the proliferation of pancreatic ductal adenocarcinoma via activating the expression of CDCA8. J Cell Physiol 2020; 235(5): 4227-4238.

12. Hayama $S$, Daigo $Y$, Yamabuki $T$, Hirata $D$, Kato $T$, Miyamoto $M$, Ito $T$, Tsuchiya $E$, Kondo $S$, Nakamura $Y$. Phosphorylation and activation of cell division cycle associated 8 by aurora kinase $B$ plays a significant role in human lung carcinogenesis. Cancer Res 2007; 67(9): 4113-4122.

13. Chang JL, Chen $T H$, Wang $C F$, Chiang $Y H$, Huang $Y L$, Wong FH, Chou CK, Chen CM. Borealin/Dasra $B$ is a cell cycle-regulated chromosomal passenger protein and its nuclear accumulation is linked to poor prognosis for human gastric cancer. Exp Cell Res 2006; 312(7): 962-973.

14. Livak KJ, Schmittgen TD. Analysis of relative gene expression data using real-time quantitative $P C R$ and the 2(-Delta Delta C(T)) Method. Methods 2001; 25(4): 402-408.

15. Singh BN, Kumar D, Shankar S, Srivastava RK. Rottlerin induces autophagy which leads to apoptotic cell death through inhibition of PI3K/Akt/mTOR pathway in human pancreatic cancer stem cells. Biochem Pharmacol 2012; 84(9): 1154-1163. 
16. Fruman DA, Rommel C. PI3K and cancer: lessons, challenges and opportunities. Nat Rev Drug Discov 2014; 13(2): 140-156.

17. Ci C, Tang B, Lyu D, Liu W, Qiang D, Ji X, Qiu X, Chen $L$, Ding $W$. Overexpression of CDCA8 promotes the malignant progression of cutaneous melanoma and leads to poor prognosis. Int J Mol Med 2019; 43(1): 404412.

18. Gao $X$, Wen $X$, He $H$, Zheng $L$, Yang $Y$, Yang J, Liu $H$, Zhou $X$, Yang $C$, Chen $Y$ et al. Knockdown of CDCA8 inhibits the proliferation and enhances the apoptosis of bladder cancer cells. PeerJ 2020; 8: e9078.

19. Yu D, Shi L, Bu Y, Li W. Cell Division Cycle Associated 8 Is a Key Regulator of Tamoxifen Resistance in Breast Cancer. J Breast Cancer 2019; 22(2): 237-247.

20. Dai C, Miao CX, Xu XM, Liu LJ, Gu YF, Zhou D, Chen LS, Lin G, Lu GX. Transcriptional activation of human CDCA8 gene regulated by transcription factor NF-Y in embryonic stem cells and cancer cells. J Biol Chem 2015; 290(37): 22423-22434.
21. Wei $W, L v Y$, Gan $Z$, Zhang $Y$, Han $X, X u Z$. Identification of key genes involved in the metastasis of clear cell renal cell carcinoma. Oncol Lett 2019; 17(5): 4321-4328.

22. Song $Z$, Huang $Y$, Zhao $Y$, Ruan $H$, Yang $H$, Cao $Q$, Liu $D$, Zhang $X$, Chen $K$. The Identification of Potential Biomarkers and Biological Pathways in Prostate Cancer. J Cancer 2019; 10(6): 1398-1408.

23. Thiery JP, Acloque H, Huang RY, Nieto MA. Epithelialmesenchymal transitions in development and disease. Cell 2009; 139(5): 871-890.

24. Huang $Y$, Zhang J, Hou L, Wang G, Liu H, Zhang $R$, Chen $X$, Zhu J. LncRNA AK023391 promotes tumorigenesis and invasion of gastric cancer through activation of the PI3K/Akt signaling pathway. J Exp Clin Cancer Res 2017; 36(1): 1-14.

25. Lee HJ, Venkatarame Gowda Saralamma V, Kim SM, Ha SE, Raha S, Lee WS, Kim EH, Lee SJ, Heo JD, Kim GS. Pectolinarigenin Induced Cell Cycle Arrest, Autophagy, and Apoptosis in Gastric Cancer Cell via PI3K/AKT/mTOR Signaling Pathway. Nutrients 2018; 10(8):1043. 